\title{
Leitura de ondas cerebrais como ferramenta para escolha das melhores práticas pedagógicas por parte dos docentes: um estudo quase experimental com estudantes do ensino superior
}

\author{
Giovanni Bohm Machado - PPGIE UFRGS/UNISINOS - giobohm@ gmail.com \\ Leandro Krug Wives - PPGIE UFRGS - leandro.wives@ufrgs.br
}

\begin{abstract}
Resumo. $O$ objetivo deste estudo foi identificar, dentre três métodos/ferramentas/formas distintas de ensino, quais despertam maior interesse e retém a atenção dos estudantes do ensino superior. Com essa finalidade, foi realizado um quase experimento com 57 estudantes de curso superior de uma Universidade privada da região metropolitana de Porto Alegre entre os anos de 2018 e 2019. Foi utilizado um aparelho medidor de ondas cerebrais chamado Mindwave NeuroSky para registrar os níveis de atenção e concentração em diferentes tipos de aulas: expositivas, vídeo e gamificadas. Após testes estatísticos de comparação entre os grupos estudados, os principais resultados indicaram maior efetividade para vídeo-aulas e aulas gamificadas, com aulas expositivas ficando em último lugar.
\end{abstract}

Palavras-chave: ondas cerebrais, aula expositiva, vídeo-aula, aula gamificada.

\section{Brainwave reading as a tool to choose best teaching practices: a quasi-experimental study with higher education students}

\begin{abstract}
The aim of this study was to identify, among three different methods/tools/forms of education, which arouse the most interest and retain the attention of higher education students. For this purpose, a quasi-experiment was conducted with 57 college students from a private university in the metropolitan region of Porto Alegre between 2018 and 2019. A brainwave equipment called Mindwave NeuroSky was used to record attention and concentration levels in different types of classes: lectures, video lessons and gamified classes. After statistical tests of comparison between the studied groups, the main results indicated greater effectiveness for video lessons and gamified classes, with lectures finishing last.
\end{abstract}

Keywords: brainwave, lecture, video lesson, gamified class.

\section{Introdução}

A contemporaneidade traz consigo a necessidade de ressignificação da escola e das relações que nelas se estabelecem. Os modelos que até então respondiam às demandas do mundo, encontram-se ultrapassados e descolados das necessidades e contextos em que vivemos. Essa quebra de paradigma diz respeito tanto às relações estabelecidas entre os sujeitos quanto às dos sujeitos com o conhecimento e com as formas de aprender (Canário, 2006; Sacristán, 2007; Bauman, 2009).

A tecnologia da informação tem crescido e se difundido de forma exponencial, modificando totalmente a interação do ser humano com todas as formas de comunicação 
(Castells, 1999), inclusive aquelas relacionadas ao aprendizado, disseminação e construção do conhecimento (Machado et al., 2018).

Segundo dados do WAS (2019), dos 7,7 bilhões de pessoas existentes no planeta, 4,4 bilhões são usuárias de internet, um crescimento de $19 \%$ em relação a 2017 , o que comprova o crescimento impressionante do acesso às tecnologias de informação e comunicação. No Brasil, os números são igualmente significativos. Dos 211 milhões de habitantes, 149 milhões são usuários de internet, com 140 milhões de usuários de redes. Em média, o brasileiro fica conectado durante 9 horas e 29 minutos por dia, sendo o segundo colocado em maior tempo de uso das redes (Was, 2019).

No âmbito da educação, as tecnologias da informação e comunicação (TIC) têm assumido um importante papel enquanto ferramentas pedagógicas que podem potencializar os processos de ensino e de aprendizagem. Assim, o papel da tecnologia no desenvolvimento dos profissionais da educação pode aprimorar o processo de formação continuada qualificando as práticas pedagógicas no contexto atual (Meirinhos, 2006).

Nesse contexto, um dos desafios em relação à construção e a utilização de tecnologias no âmbito educacional é de estimular os estudantes a se envolverem e buscar maior engajamento nas atividades promovidas nos ambientes destinados ao ensino e a aprendizagem (Herpich et al., 2018).

Cada vez mais a tecnologia tem revolucionado as formas de comunicação e proporcionado novas experiências de aprendizado e interação entre pares, fazendo com que as práticas pedagógicas desenvolvidas em sala de aula sejam disseminadas e compartilhadas de uma forma ampla e irrestrita (Machado, Wives e Grandi, 2019). No entanto, ainda se necessita de mais estudos relacionados as tecnologias utilizadas para o processo de ensino-aprendizagem e sua efetiva aplicação em sala de aula, ambientes virtuais de aprendizagem ou outras formas existentes.

O objetivo desde estudo é identificar, dentre três métodos/ferramentas/formas distintas de ensino, quais despertam maior interesse e retém a atenção dos estudantes do ensino superior. Especificamente, espera-se medir o nível de atenção desses estudantes em cada uma destas formas de ensino, o que pode gerar implicações importantes para $o$ desenvolvimento das práticas pedagógicas por parte dos professores.

Este documento está organizado da seguinte maneira. Primeiramente, são apresentados conceitos e fundamentos relacionados a atividades e didática de ensino em sala de aula, objetos de aprendizagem e gamificação. Em seguida, está descrita a metodologia de pesquisa, a apresentação e análise dos dados coletados. Por fim, são apresentadas as considerações finais.

\section{Marco teórico: métodos de ensino praticados no ensino superior}

O modelo mais difundido de educação tradicional são as chamadas aulas expositivas. É muito comum encontrar currículos e organização de disciplinas que acabam por incentivar, mesmo que veladamente, que os docentes utilizem aulas meramente expositivas (Anastasiou e Alves, 2010). O primeiro elemento que identifica um método de ensino, é a ordem em que se propõe as atividades, sendo a exposição dos conteúdos pelo professor, a forma praticamente dominante neste estilo (Zabala, 2010).

Do ponto de vista dos estudantes, a expectativa é receber este modelo tradicional de ensino, fato que gera acomodação, já que outra proposta pode ser difícil, dispendiosa, muito mais complicada de se obter êxito (Zabalza, 2004; Soares e Cunha, 2010; Meyer e Vosgerau, 2016). 
Os estilos de aprendizagem refletem a maneira como os estudantes percebem e processam as informações e os estímulos motivacionais que se manifestam durante o processo de conhecimento (Belhot, 1997). É preciso fazer uso dessa informação para melhor compreender as pessoas e suas necessidades; principalmente o professor quando vai preparar sua aula (Trevelin, Pereira e Oliveira Neto, 2013).

Dentro dessa premissa, surgiram os objetos de aprendizagem, também conhecidos por recursos educacionais. Os objetos de aprendizagem (OA) constituem um paradigma que busca viabilizar a reutilização de recursos educacionais digitais nos mais diversos cenários de aprendizagem (Schmitt et al., 2013). Especificamente, no âmbito deste estudo, verifica-se a importância das vídeo-aulas para o sistema de ensino moderno. $\mathrm{O}$ recurso de vídeo é uma forma de comunicação que emprega a linguagem direta e coloquial, se comunica através de emoções e amplifica o efeito de presença mediante sua distribuição (Cabral Júnior, Carneiro e Zaro, 2016). Os recursos de vídeo funcionam como instrumento de apoio aos processos de ensino e de aprendizagem, e com o advento dos cursos online se tornaram algo recorrente na educação de adultos (Catelli, 2003).

Outra forma de forma de ensino bastante utilizada nos dias de hoje é a gamificação. Segundo Fardo (2013), a gamificação pressupõe a utilização de elementos encontrados nos games, tais como narrativa, recompensas, disputas, competição, objetivos e regras definidas entre outros, com a finalidade de tentar obter o mesmo grau de envolvimento e motivação que normalmente encontramos nos jogadores quando em interação com bons games.

Todos estes métodos e ferramentas aqui apresentadas serão objeto de estudo deste artigo, cuja metodologia será detalhada na próxima seção.

\section{Metodologia}

Para este estudo, será utilizada a técnica do quase experimento para identificar, dentre três métodos/ferramentas/formas distintas de ensino, quais despertam maior interesse e retém a atenção dos estudantes do ensino superior.

A pesquisa foi realizada com turmas de uma mesma disciplina da área de Gestão e Negócios de uma Universidade privada da região metropolitana de Porto Alegre. Foram selecionados, a priori, 59 estudantes oriundos de turmas pertencentes aos semestres 2018/1, 2018/2 e 2019/1. É importante salientar de que 100\% dos estudantes matriculados nas respectivas turmas aceitaram participar do processo quase experimental, preenchendo e assinando um Termo de Consentimento e Livre Esclarecido tomando ciência dos objetivos da pesquisa e autorizando a utilização dos dados coletados.

A coleta de dados foi realizada nos meses de abril de 2018, novembro de 2018 e maio de 2019, nas dependências da Universidade em uma sala reservada, dotada de quadro branco e um computador. Antes da aplicação do quase experimento, cada estudante respondeu 3 perguntas básicas, idade, gênero e tempo de estudo na Universidade. Optou-se pela coleta desses dados para robustecer as informações colhidas e para que no futuro, possam ser utilizados em estudos complementares.

Foi utilizado um aparelho de leitura de ondas cerebrais chamado Mindwave NeuroSky, que mede o estado de atenção e concentração do usuário. Os dados são monitorados e gravados por um aplicativo de smartphone chamado Effective Learner, produzido pela fabricante do aparelho. Cada estudante foi testado individualmente. $\mathrm{O}$ aparelho, uma espécie de tiara, foi colocado na cabeça de cada um dos estudantes, onde foi posicionado o leitor de ondas cerebrais na região da testa (lóbulo frontal) e uma presilha na orelha direita (medição de batimentos cardíacos).

V. $17 \mathrm{~N}^{\circ}$ 3, dezembro, 2019

RENOTE DOI: 
Todos os estudantes da turma 2018/1, de forma individual, receberam uma aula expositiva diretamente de um professor, com duração de 3 minutos sobre um assunto préselecionado. Enquanto a aula acontecia, os dados oriundos do Mindwave NeuroSky estavam sendo gravados por um dispositivo smartphone através do aplicativo Effective Learner.

Os estudantes da turma 2018/2, também de forma individual, acessaram a Plataforma Moodle da Universidade para assistir a uma vídeo-aula, com exatamente o mesmo conteúdo aplicado na aula expositiva e pelo mesmo professor. A duração foi idêntica ao da aula expositiva, 3 minutos. Os dados também foram gravados enquanto os estudantes assistiam ao vídeo.

Os estudantes da última turma, de 2019/1, individualmente, acessaram a uma plataforma chamada Kahoot, onde puderam acessar um conteúdo gamificado de igual teor às turmas anteriores, desenvolvido pelo mesmo professor. A duração do exercício também durou aproximadamente 3 minutos e os dados de efetividade foram igualmente gravados como nos casos anteriores.

Foram testados 19 estudantes da turma 2018/1,21 da turma 2018/2 e 19 da turma 2019/1. As informações colhidas pelo aplicativo Effective Learner foram tabuladas em uma tabela no Microsoft Excel para posterior análise. Essas informações mostram graficamente, seis estados de atenção e concentração, sendo 3 "menos efetivos" e 3 "mais efetivos". Após a aplicação dos testes, foi decidido retirar 2 estudantes da turma 2018/2, a fim de que o número amostral fosse o mesmo para todas as turmas. Foram retirados os estudantes com maior e o menor resultado.

Pode-se verificar um exemplo de como as informações são visualizadas no aplicativo Effective Learner, conforme Figura 1.

Figura 1. Exemplo de tela de resultados do aplicativo Effective Learner

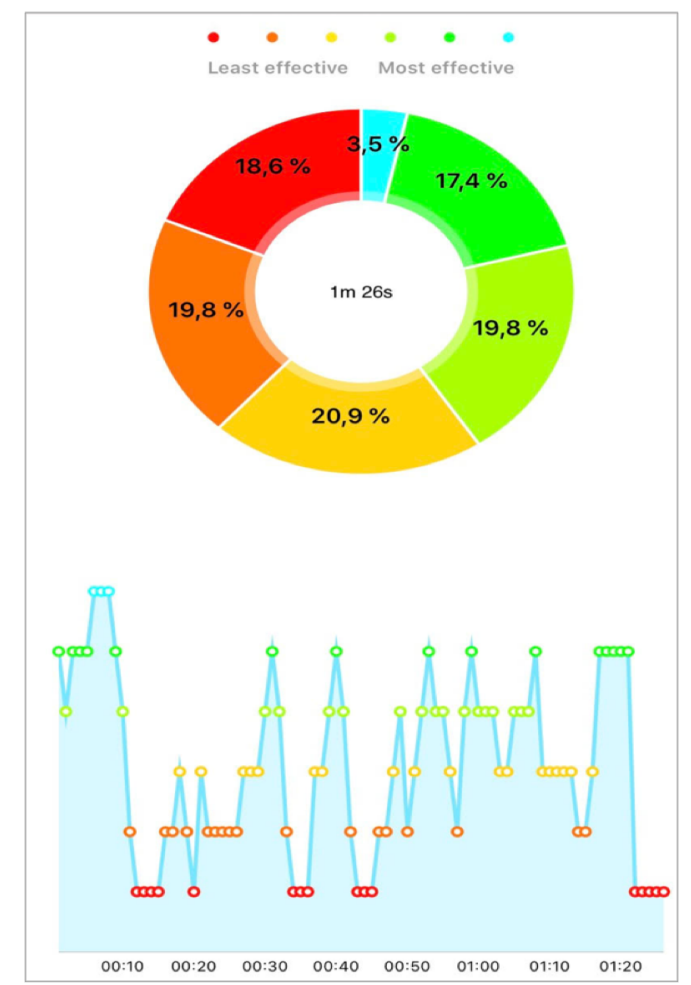

Na próxima seção, serão apresentados os dados coletados bem como as análises réahiardsdezembro, 2019 RENOTE DOI: 


\section{Apresentação e Análise dos dados}

A fim de caracterizar de forma básica os respondentes desta pesquisa, foram criadas 3 questões pontuais, como faixa etária, gênero e tempo em que estão estudando na instituição de ensino superior. As informações foram separadas por grupo e os dados podem ser verificados na Tabela 1 .

Tabela 1. Caracterização da amostra

\begin{tabular}{|c|c|c|c|c|}
\hline \multirow{2}{*}{$\#$} & \multirow{2}{*}{ Descrição } & Grupo 1 & Grupo 2 & Grupo 3 \\
\hline & & Expositiva & Vídeo-aula & Gamificação \\
\hline \multirow{4}{*}{ 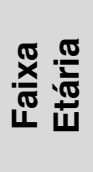 } & 18 a 24 anos & $10 \%$ & $12 \%$ & $9 \%$ \\
\hline & 24 a 29 anos & $58 \%$ & $52 \%$ & $47 \%$ \\
\hline & 30 a 39 anos & $30 \%$ & $33 \%$ & $40 \%$ \\
\hline & 40 a 59 anos & $2 \%$ & $3 \%$ & $4 \%$ \\
\hline \multirow{3}{*}{ 잏 } & Masculino & $44 \%$ & $48 \%$ & $45 \%$ \\
\hline & Feminino & $56 \%$ & $51 \%$ & $55 \%$ \\
\hline & Outro & $0 \%$ & $1 \%$ & $0 \%$ \\
\hline \multirow{5}{*}{$\begin{array}{l}\text { 을 음 } \\
\text { 들 } \\
\text { 늠 }\end{array}$} & menos de 1 ano & $1 \%$ & $2 \%$ & $1 \%$ \\
\hline & 1 a 2 anos & $3 \%$ & $3 \%$ & $4 \%$ \\
\hline & 2 a 3 anos & $47 \%$ & $58 \%$ & $51 \%$ \\
\hline & 3 a 4 anos & $34 \%$ & $24 \%$ & $40 \%$ \\
\hline & mais de 4 anos & $15 \%$ & $13 \%$ & $4 \%$ \\
\hline
\end{tabular}

Depois de caracterizar a amostra, o desafio foi atribuir valores numéricos aos percentuais de efetividade obtidos para cada um dos sujeitos da pesquisa. A solução encontrada, foi atribuir um peso relativo à da camada de efetividade medida pelo aplicativo, variando do numeral 1 (um) para o menos efetivo até o numeral 6 (seis) para o mais efetivo. Foi definido que para cada variação de efetividade, o percentual deveria ser multiplicado pelo número correspondente e somado aos demais, resultando em um número inteiro que pudesse expressar a média de efetividade de cada sujeito, conforme disposto na Figura 2.

Figura 2. Fórmula para cálculo da média de efetividade de cada sujeito

$$
\text { Resultado }=(v 1 * 1)+(v 2 * 2)+(v 3 * 3)+(v 4 * 4)+(v 5 * 5)+(v 6 * 6)
$$

Os valores v1, v2 e v3 correspondem aos percentuais dos resultados menos efetivos, enquanto os valores $\mathrm{v} 4$, v5 e v6 correspondem aos percentuais dos resultados mais efetivos. Tal referência de efetividade foi apresentada no exemplo da Figura 1. Na Tabela 2 são apresentados os valores obtidos e os resultados calculados para cada sujeito participante da pesquisa.

Tabela 2. Resultado das atribuições de peso aos percentuais de efetividade

\begin{tabular}{c|c|r|c|c|c|c|c|c}
\hline \multirow{2}{*}{$\#$} & \multirow{2}{*}{ Grupo } & \multicolumn{3}{|c|}{ Menos efetivo } & \multicolumn{2}{|c|}{ Mais efetivo } & \multirow{2}{*}{ Resultado } \\
\cline { 3 - 8 } & & v1 & v2 & v3 & v4 & v5 & v6 & \\
\hline Sujeito 1 & Aula expositiva & $10 \%$ & $12 \%$ & $33 \%$ & $31 \%$ & $11 \%$ & $3 \%$ & 3 \\
\hline Sujeito 2 & Aula expositiva & $9 \%$ & $13 \%$ & $28 \%$ & $33 \%$ & $9 \%$ & $8 \%$ & 3 \\
\hline Sujeito 3 & Aula expositiva & $11 \%$ & $11 \%$ & $20 \%$ & $37 \%$ & $14 \%$ & $7 \%$ & 4 \\
\hline Sujeito 4 & Aula expositiva & $8 \%$ & $42 \%$ & $29 \%$ & $11 \%$ & $4 \%$ & $6 \%$ & 3 \\
\hline
\end{tabular}




\begin{tabular}{|c|c|c|c|c|c|c|c|c|}
\hline \multirow{2}{*}{ \# } & \multirow{2}{*}{ Grupo } & \multicolumn{3}{|c|}{ Menos efetivo } & \multicolumn{3}{|c|}{ Mais efetivo } & \multirow{2}{*}{ Resultado } \\
\hline & & v1 & v2 & v3 & v4 & v5 & v6 & \\
\hline Sujeito 5 & Aula expositiva & $12 \%$ & $13 \%$ & $14 \%$ & $41 \%$ & $13 \%$ & $7 \%$ & 4 \\
\hline Sujeito 6 & Aula expositiva & $9 \%$ & $7 \%$ & $9 \%$ & $8 \%$ & $32 \%$ & $35 \%$ & 5 \\
\hline Sujeito 7 & Aula expositiva & $36 \%$ & $40 \%$ & $7 \%$ & $5 \%$ & $5 \%$ & $7 \%$ & 2 \\
\hline Sujeito 8 & Aula expositiva & $18 \%$ & $21 \%$ & $38 \%$ & $7 \%$ & $11 \%$ & $5 \%$ & 3 \\
\hline Sujeito 9 & Aula expositiva & $8 \%$ & $7 \%$ & $17 \%$ & $44 \%$ & $15 \%$ & $9 \%$ & 4 \\
\hline Sujeito 10 & Aula expositiva & $16 \%$ & $12 \%$ & $37 \%$ & $15 \%$ & $11 \%$ & $5 \%$ & 3 \\
\hline Sujeito 11 & Aula expositiva & $19 \%$ & $28 \%$ & $23 \%$ & $18 \%$ & $10 \%$ & $2 \%$ & 3 \\
\hline Sujeito 12 & Aula expositiva & $12 \%$ & $17 \%$ & $43 \%$ & $14 \%$ & $9 \%$ & $5 \%$ & 3 \\
\hline Sujeito 13 & Aula expositiva & $9 \%$ & $11 \%$ & $5 \%$ & $67 \%$ & $6 \%$ & $2 \%$ & 4 \\
\hline Sujeito 14 & Aula expositiva & $29 \%$ & $39 \%$ & $7 \%$ & $12 \%$ & $6 \%$ & $7 \%$ & 2 \\
\hline Sujeito 15 & Aula expositiva & $8 \%$ & $8 \%$ & $9 \%$ & $10 \%$ & $31 \%$ & $34 \%$ & 5 \\
\hline Sujeito 16 & Aula expositiva & $15 \%$ & $11 \%$ & $37 \%$ & $21 \%$ & $8 \%$ & $8 \%$ & 3 \\
\hline Sujeito 17 & Aula expositiva & $18 \%$ & $15 \%$ & $32 \%$ & $19 \%$ & $7 \%$ & $9 \%$ & 3 \\
\hline Sujeito 18 & Aula expositiva & $14 \%$ & $14 \%$ & $49 \%$ & $11 \%$ & $10 \%$ & $2 \%$ & 3 \\
\hline Sujeito 19 & Aula expositiva & $11 \%$ & $15 \%$ & $53 \%$ & $13 \%$ & $7 \%$ & $1 \%$ & 3 \\
\hline Sujeito 20 & Vídeo-aula & $12 \%$ & $11 \%$ & $19 \%$ & $35 \%$ & $18 \%$ & $5 \%$ & 4 \\
\hline Sujeito 21 & Vídeo-aula & $19 \%$ & $33 \%$ & $32 \%$ & $7 \%$ & $5 \%$ & $4 \%$ & 3 \\
\hline Sujeito 22 & Vídeo-aula & $5 \%$ & $36 \%$ & $25 \%$ & $18 \%$ & $9 \%$ & $7 \%$ & 3 \\
\hline Sujeito 23 & Vídeo-aula & $7 \%$ & $10 \%$ & $14 \%$ & $36 \%$ & $23 \%$ & $10 \%$ & 4 \\
\hline Sujeito 24 & Vídeo-aula & $1 \%$ & $6 \%$ & $15 \%$ & $11 \%$ & $49 \%$ & $18 \%$ & 5 \\
\hline Sujeito 25 & Vídeo-aula & $4 \%$ & $5 \%$ & $11 \%$ & $13 \%$ & $44 \%$ & $23 \%$ & 5 \\
\hline Sujeito 26 & Vídeo-aula & $4 \%$ & $7 \%$ & $11 \%$ & $12 \%$ & $43 \%$ & $23 \%$ & 5 \\
\hline Sujeito 27 & Vídeo-aula & $5 \%$ & $9 \%$ & $12 \%$ & $11 \%$ & $31 \%$ & $32 \%$ & 5 \\
\hline Sujeito 28 & Vídeo-aula & $8 \%$ & $17 \%$ & $13 \%$ & $21 \%$ & $35 \%$ & $6 \%$ & 4 \\
\hline Sujeito 29 & Vídeo-aula & $9 \%$ & $7 \%$ & $14 \%$ & $18 \%$ & $37 \%$ & $15 \%$ & 4 \\
\hline Sujeito 30 & Vídeo-aula & $4 \%$ & $11 \%$ & $9 \%$ & $10 \%$ & $34 \%$ & $32 \%$ & 5 \\
\hline Sujeito 31 & Vídeo-aula & $10 \%$ & $8 \%$ & $13 \%$ & $45 \%$ & $21 \%$ & $3 \%$ & 4 \\
\hline Sujeito 32 & Vídeo-aula & $8 \%$ & $35 \%$ & $21 \%$ & $20 \%$ & $8 \%$ & $8 \%$ & 3 \\
\hline Sujeito 33 & Vídeo-aula & $3 \%$ & $12 \%$ & $8 \%$ & $12 \%$ & $35 \%$ & $30 \%$ & 5 \\
\hline Sujeito 34 & Vídeo-aula & $9 \%$ & $7 \%$ & $11 \%$ & $54 \%$ & $15 \%$ & $4 \%$ & 4 \\
\hline Sujeito 35 & Vídeo-aula & $7 \%$ & $11 \%$ & $7 \%$ & $13 \%$ & $45 \%$ & $17 \%$ & 4 \\
\hline Sujeito 36 & Vídeo-aula & $2 \%$ & $7 \%$ & $11 \%$ & $11 \%$ & $54 \%$ & $15 \%$ & 5 \\
\hline Sujeito 37 & Vídeo-aula & $4 \%$ & $8 \%$ & $11 \%$ & $7 \%$ & $41 \%$ & $29 \%$ & 5 \\
\hline Sujeito 38 & Vídeo-aula & $2 \%$ & $2 \%$ & $6 \%$ & $16 \%$ & $56 \%$ & $18 \%$ & 5 \\
\hline Sujeito 39 & Gamificação & $13 \%$ & $21 \%$ & $29 \%$ & $23 \%$ & $9 \%$ & $5 \%$ & 3 \\
\hline Sujeito 40 & Gamificação & $18 \%$ & $3 \%$ & $35 \%$ & $31 \%$ & $11 \%$ & $2 \%$ & 3 \\
\hline Sujeito 41 & Gamificação & $14 \%$ & $19 \%$ & $37 \%$ & $18 \%$ & $3 \%$ & $9 \%$ & 3 \\
\hline Sujeito 42 & Gamificação & $16 \%$ & $22 \%$ & $34 \%$ & $19 \%$ & $6 \%$ & $3 \%$ & 3 \\
\hline Sujeito 43 & Gamificação & $17 \%$ & $14 \%$ & $45 \%$ & $21 \%$ & $2 \%$ & $1 \%$ & 3 \\
\hline Sujeito 44 & Gamificação & $9 \%$ & $12 \%$ & $17 \%$ & $34 \%$ & $21 \%$ & $7 \%$ & 4 \\
\hline
\end{tabular}




\begin{tabular}{c|c|r|r|r|r|r|c|c}
\hline \multirow{2}{*}{$\#$} & \multirow{2}{*}{ Grupo } & \multicolumn{3}{|c|}{ Menos efetivo } & \multicolumn{2}{|c|}{ Mais efetivo } & \multirow{2}{*}{ Resultado } \\
\cline { 3 - 7 } & & v1 & v2 & v3 & v4 & v5 & v6 & \\
\hline \hline Sujeito 45 & Gamificação & $14 \%$ & $10 \%$ & $18 \%$ & $32 \%$ & $19 \%$ & $7 \%$ & 4 \\
\hline Sujeito 46 & Gamificação & $7 \%$ & $13 \%$ & $19 \%$ & $29 \%$ & $19 \%$ & $13 \%$ & 4 \\
\hline Sujeito 47 & Gamificação & $9 \%$ & $7 \%$ & $14 \%$ & $47 \%$ & $11 \%$ & $12 \%$ & 4 \\
\hline Sujeito 48 & Gamificação & $11 \%$ & $15 \%$ & $11 \%$ & $50 \%$ & $9 \%$ & $14 \%$ & 4 \\
\hline Sujeito 49 & Gamificação & $11 \%$ & $3 \%$ & $5 \%$ & $7 \%$ & $53 \%$ & $21 \%$ & 5 \\
\hline Sujeito 50 & Gamificação & $1 \%$ & $1 \%$ & $3 \%$ & $21 \%$ & $53 \%$ & $21 \%$ & 5 \\
\hline Sujeito 51 & Gamificação & $8 \%$ & $3 \%$ & $7 \%$ & $10 \%$ & $57 \%$ & $15 \%$ & 5 \\
\hline Sujeito 52 & Gamificação & $13 \%$ & $11 \%$ & $12 \%$ & $38 \%$ & $21 \%$ & $5 \%$ & 4 \\
\hline Sujeito 53 & Gamificação & $9 \%$ & $10 \%$ & $16 \%$ & $41 \%$ & $17 \%$ & $7 \%$ & 4 \\
\hline Sujeito 54 & Gamificação & $18 \%$ & $21 \%$ & $48 \%$ & $7 \%$ & $1 \%$ & $5 \%$ & 3 \\
\hline Sujeito 55 & Gamificação & $10 \%$ & $16 \%$ & $37 \%$ & $21 \%$ & $7 \%$ & $9 \%$ & 3 \\
\hline Sujeito 56 & Gamificação & $26 \%$ & $8 \%$ & $35 \%$ & $19 \%$ & $11 \%$ & $1 \%$ & 3 \\
\hline Sujeito 57 & Gamificação & $11 \%$ & $26 \%$ & $40 \%$ & $12 \%$ & $7 \%$ & $4 \%$ & 3 \\
\hline \hline
\end{tabular}

O teste de normalidade resultou em valor p $>0.05$ tanto para Kolmogorov-Smirnov quanto para Shapiro-Wilk, o que indica a normalidade da amostra coletada (Hair et al., 2005), conforme Tabela 3.

Tabela 3. Teste de normalidade da amostra

\begin{tabular}{c|c|c|c|c|c}
\hline \multicolumn{2}{c|}{ Kolmogorov-Smirnov } & \multicolumn{3}{c}{ Shapiro-Wilk } \\
\hline Estatística & gl & Sig. & Estatística & gl & Sig. \\
\hline, 090 & 57 &, 200 &, 978 & 57 &, 770 \\
\hline
\end{tabular}

O teste de Levene, que mede a homogeneidade de variâncias da amostra, indicou valor $p=0.999$, o que indica a que a amostra coletada varia de forma homogênea, conforme pode ser verificado na Tabela 4.

Tabela 4. Homogeneidade de variâncias

\begin{tabular}{c|c|c|c}
\hline Teste de Levene & gl1 & gl2 & Sig. \\
\hline \hline, 001 & 2 & 54 &, 999 \\
\hline
\end{tabular}

O teste ANOVA de um fator foi aplicado para comparação dos 3 grupos de alunos testados. Valores de $p$ iguais ou menores 0.05 , indicam diferença entre os grupos e os valores maiores de 0.05 indicam que não existe diferença entre os grupos (Hair et al., 2005).

O teste indicou que existe diferença entre os grupos, com valor $p=0.001$, de acordo com a Tabela 5.

Tabela 5. Teste ANOVA de um fator

\begin{tabular}{l|c|c|c|c|c}
\hline Relações & $\begin{array}{c}\text { Soma dos } \\
\text { Quadrados }\end{array}$ & gl & $\begin{array}{c}\text { Quadrado } \\
\text { Médio }\end{array}$ & F & Sig. \\
\hline \hline Entre Grupos & 9,719 & 2 & 4,860 & 8,121 &, 001 \\
Nos grupos & 32,316 & 54 &, 598 & & \\
Total & 42,035 & 56 & & & \\
\hline
\end{tabular}

V. 17 No 3, dezembro, 2019 DOI: 
Uma vez significante a diferença entre os grupos, é preciso analisar quais deles diferem-se entre si e quais as implicações estatísticas e práticas dessas diferenças. Para tal, foram aplicados os testes de Tukey e Scheffe, conforme Tabela 6.

Tabela 6. Especificação das diferenças entre os grupos (post-hoc)

\begin{tabular}{|c|c|c|c|c|c|}
\hline Teste & (I) Grupo & (J) Grupo & $\begin{array}{c}\text { Diferença média } \\
(I-J)\end{array}$ & $\begin{array}{c}\text { Erro } \\
\text { Padrão }\end{array}$ & Sig. \\
\hline \multirow{6}{*}{ Tukey HSD } & \multirow{2}{*}{ Expositivas } & Vídeo-aula & $-1,00000$ & "25099 & "001 \\
\hline & & Gamificação & -,36842 & ,25099 & ,314 \\
\hline & \multirow{2}{*}{ Vídeo-aula } & Expositivas & 1,00000 & ,25099 & 001 \\
\hline & & Gamificação & ,63158 & ,25099 &, 039 \\
\hline & \multirow{2}{*}{ Gamificação } & Expositivas & ,36842 & ,25099 & ,314 \\
\hline & & Vídeo-aula &,- 63158 & ,25099 &, 039 \\
\hline \multirow{6}{*}{ Scheffe } & \multirow{2}{*}{ Expositivas } & Vídeo-aula & $-1,00000$ & ,25099 & , 001 \\
\hline & & Gamificação &,- 36842 & ,25099 & ,348 \\
\hline & \multirow{2}{*}{ Vídeo-aula } & Expositivas & 1,00000 & ,25099 &, 001 \\
\hline & & Gamificação & ,63158 & ,25099 &, 050 \\
\hline & \multirow{2}{*}{ Gamificação } & Expositivas & ,36842 & ,25099 & ,348 \\
\hline & & Vídeo-aula & -,63158 & 25099 & ,050 \\
\hline
\end{tabular}

Lembrando que, para análise dos grupos post-hoc, os valores iguais ou menores 0.05 indicam diferença entre os outros e os valores maiores de 0.05 indicam que não existe diferença entre os grupos comparados (Hair et al., 2005).

Os dados da Tabela 6 mostram efetivamente as diferenças estatísticas entre os grupos pesquisados. Tanto no teste de Tukey como no teste de Scheffe, os resultados em termos de significância são os mesmos. Para fins de análise, serão descritos os valores do teste de Tukey.

Quando comparado o grupo que recebeu aulas expositivas e o grupo que recebeu vídeo aulas, foi constatada diferença entre os grupos, com $p=0.001$. Já em relação a gamificação, não há diferença estatística entre aulas expositivas e a aula gamificada $p=0.314$.

Na comparação entre aula gamificada e vídeo-aula, é possível indicar que não há diferença estatística entre os grupos, $\operatorname{com} p=0,39$.

Com base nas médias dos resultados dos grupos, constantes na Tabela 2, e nos resultados das análises comparativas entre os grupos, é possível afirmar que a utilização de vídeo-aulas e aulas gamificadas tem melhor resultados para com os estudantes em termos de fixação da atenção e maior concentração, conforme Tabela 7.

Tabela 7. Média geral dos resultados

\begin{tabular}{l|c|c|c}
\hline Método & Sujeitos & Média inteira & Média real \\
\hline \hline Aula expositiva & 19 & 3 & 3,23 \\
\hline Vídeo-aula & 19 & 4 & 4,04 \\
\hline Gamificação & 19 & 4 & 3,51 \\
\hline
\end{tabular}


A média real, indica que o modelo da vídeo-aula foi mais efetivo nos testes do que a aula gamificada, com valores de 4,04 e 3,51 respectivamente. Definitivamente, a aula expositiva foi a que obteve o pior resultado perante os estudantes, com média real de 3,23.

\section{Considerações Finais}

Este estudo buscou identificar, dentre três formas distintas de ensino, quais despertam maior interesse e retém a atenção dos estudantes do ensino superior. Foi possível medir, através de um quase experimento e com o auxílio de um dispositivo eletrônico para leitura de ondas cerebrais, o nível de atenção de estudantes de uma Universidade privada em relação a aulas expositivas, vídeo-aulas e aula gamificada.

Os principais resultados indicaram a existência de diferença estatística entre os grupos pesquisados, mais especificamente entre aulas expositivas e os demais métodos. Os testes indicaram vantagens da vídeo-aula e da aula gamificada em relação ao método tradicional da aula expositiva. Ainda foi possível verificar que o modelo da vídeo-aula leva vantagem em relação a aula gamificada, possuindo média geral superior.

Através deste estudo, foi possível confirmar de que tecnologias capazes de estimular os estudantes são capazes de tornar o processo de ensino-aprendizagem mais atrativo para esse público (Herpich et al., 2018).

A evolução constante da tecnologia tem proporcionado com que novas práticas pedagógicas estejam sendo desenvolvidas e disseminadas para uma ampla gama de pessoas (Machado, Wives e Grandi, 2019), o que favorece a utilização de práticas com a vídeo-aula e recursos gamificados (Fardo, 2013; Cabral Júnior, Carneiro e Zaro, 2016).

Como proposta de trabalhos futuros, propõe-se a testagem de um número maior de pessoas e turmas de diferentes áreas de atuação, bem como a experimentação de novos modelos pedagógicos como aulas invertidas entre outros.

\section{Referências}

ANAStasiou, L. G. C; ALVES, L. P. Processos de ensino na universidade: pressupostos e estratégias de trabalho em sala de aula. Joinville: Univille, 2010.

BAUMAN, Z. Entrevista sobre educação, desafios pedagógicos e modernidade líquida. Cadernos de Pesquisa. v.39, n.137, pp. 661-684, 2009.

BELHOT, R. V. Reflexões e Propostas sobre o "Ensinar Engenharia para o Século XXI".Tese de Livre - Docência, Engenharia, São Carlos - SP, USP - Universidade de São Paulo, 1997.

CABRAL JUNIOR, P. A. F., CARNEIRO, M. L. F. ZARO, M. A. Influências da interação entre professor e o processo de produção audiovisual no resultado final de vídeos educacionais. RENOTE - Revista de Novas Tecnologias na Educação. V. $14 \mathrm{~N}^{\mathrm{o}} 2,2016$.

CANÁRIO, R. A escola tem futuro? Das promessas às incertezas. Porto Alegre: Artmed, 2006.

CASTELLS, M. A sociedade em rede. A era da Informação: Economia. Sociedade e Cultura, v. 1, p. 5, 1999.

CATELli, R. E. Cinema e Educação em John Grierson. 2003. Disponível em: $<$ http://www.mnemocine.com.br/aruanda/cineducemgrierson.htm >. Acesso em 19 de outubro de 2019. 
FARDO, M. L. A gamificação aplicada em ambientes de aprendizagem. RENOTE Revista de Novas Tecnologias na Educação. V. 11 N$^{\circ} 1,2013$.

HERPICH, F. BOS, A. KUHN, I. GUARESE, R. L. M., TAROUCO, L. M. R., WIVES, L. K., ZARO, M. A. Atividade cerebral no uso de recursos educacionais em realidade aumentada: uma análise da atenção do aprendiz. Anais do XXIX Simpósio Brasileiro de Informática na Educação - SBIE, 2018.

MACHADO, G. B., KUHN, I. SANTOS JR. F. D, WIVES, L. K. Um Estudo Sobre o Perfil de Professores do Ensino Fundamental e o Uso de Tecnologias para a Educação: uma Proposição de Agenda de Pesquisa a partir de Dados Educacionais. RENOTE Revista de Novas Tecnologias na Educação, v. 16, n. 2, 2018.

MACHADO, G. B., WIVES, L. K., GRANDI, R. As comunidades de prática como ferramenta para formação continuada de professores: percepções docentes sobre o uso da tecnologia para compartilhamento e aprimoramento das práticas pedagógicas. Anais do XXX Simpósio Brasileiro de Informática na Educação - SBIE, 2019.

MEIRINHOS, M. F. A. Desenvolvimento profissional docente em ambientes colaborativos de aprendizagem a distância: estudo de caso no âmbito da formação contínua. 2006. 362 f. Tese (Doutorado) - Instituto de Estudos da Criança, Universidade do Minho, Braga, 2006.

MEYER, P. et. al. Inquietudes pedagógicas: os professores universitários na superação do modelo tradicional. Anais da Reunião Científica Regional da ANPED Educação, movimentos sociais e políticas governamentais. UPFR - Curitiba/PR. 24 a 27 de julho, 2016.

SACRISTÁN, J. G. Consciência e acção sobre a prática como libertação profissional dos professores. IN: NÓVOA, Antônio. Profissão professor. Porto Editora: Portugal, 1991.

SCHMITT, M. A. R., TAROUCO, L. M. R., RODRIGUES, A. P., VIDEIRA, J. A. Depósito de objetos de aprendizagem em repositórios a partir da integração com ambientes virtuais de aprendizagem. RENOTE - Revista de Novas Tecnologias na Educação. V. $11 \mathrm{~N}^{\mathrm{o}}$ 3, 2013.

TARDIF, M. Saberes docentes e formação profissional. Petrópolis, RJ: Vozes, 2002.

TREVELIN, A. T. C., PEREIRA, M. A. A., OLIVEIRA NETO, J. D. "A utilização da "sala de aula invertida" em cursos superiores de tecnologia: comparação entre o modelo tradicional e o modelo invertido "flipped classroom" adaptado aos estilos de aprendizagem." Revista de estilos de aprendizaje 6.12, 2013.

WAS, We Are Social. Digital 2019 Global Digital Overview. Disponível em https://www.slideshare.net/DataReportal/digital-2019-global-digital-overviewjanuary-2019-v01?from_action=save Acessado em 21/06/2019.

ZABALA, A. A prática educativa: como ensinar. Porto Alegre: Artmed, 2010.

ZABALZA, M. A. O ensino universitário: seu cenário, seus protagonistas. Porto Alegre: Artmed, 2004. 